\title{
Superfluid helium-4 hydrodynamics with discrete topological defects
}

\author{
Demosthenes Kivotides \\ University of Strathclyde Glasgow
}

(Dated: September 16, 2018)

\begin{abstract}
In superfluid helium-4, a model of normal-fluid hydrodynamics and their coupling with topological defects (quantized vortices) of the order parameter (superfluid) is formulated. The model requires only material properties as input, and applies to both laminar and turbulent flows, to both dilute and dense superfluid vortex tangles. By solving the model for the case of a normal-fluid vorticity Hopf-link interacting with systems of quantized vortices, two vortex dynamical mechanisms of energy transfer from the normal-fluid to the superfluid are indicated: (a) small superfluid rings expand to the size of the normal-fluid vortex link tubes, and (b) superfluid rings with diameters similar to the diameters of the normal-fluid tubes succumb to axial-flow instabilities that excite small amplitude wiggles which subsequently evolve into spiral-waves along the superfluid vortex contours. The normal-fluid vorticity scale determines the upper size of the generated superfluid vorticity structures. A key role in energy transfer processes is played by an axial-flow instability of a superfluid vortex due to mutual-friction excitation by the normal-fluid, which mirrors the instability of normal-fluid tubes due to mutual-friction excitation by the superfluid. Although the sites of superfluid vorticity generation are always in the neighbourhood of intense normal-fluid vorticity events, the superfluid vortices do not mimic the normal-fluid vorticity structure, and perform different motions. These vortex dynamical processes provide explanations for the phenomenology of fully developed finite temperature superfluid turbulence.
\end{abstract}




\section{PROLOGUE}

Due to quantum decoherence and the loss of quantum interference effects $[1,2]$, the hydrodynamics of many quantum systems (e.g., quark-gluon plasmas [3] or helium-4 liquids above the critical temperature of $T=2.17 \mathrm{~K}$ ) follow similar equations with those that apply to classical gases and liquids. In the helium- 4 case however, below $T=2.17 \mathrm{~K}$ (the so-called lambda point), the global $U(1)$ symmetry of the microscopic quantum system is spontaneously broken (Bose-Einstein Condensation), and low-frequency, long-wavelength Nambu-Goldstone modes appear, that need vanishingly little energy to excite, and are referred to as order-parameter dynamics [4]. These modes are of different nature than the (normal-fluid) hydrodynamics corresponding to conservation laws. Although spontaneous symmetry breaking is also a feature of classical systems (e.g., topological defect networks in liquid crystals: Poiseuille flow [5] or simple shear flow [6]), in helium-4, the broken symmetry corresponds to the conservation of particle number, and the corresponding order parameter obeys a nonlinear Schroedinger equation that depicts an inviscid, compressible superfluid populated with topological defects (vortices). In other words, the order parameter is a $m a-$ terial field, a rather intriguing physics case. The term material field indicates that, rather than been (for example) a quality like the net magnetization in a ferromagnetic system undergoing a phase transition, the superfluid order parameter corresponds to the density and momentum of matter. The topological defects are real-life examples of the line-vortices of inviscid hydrodynamics, albeit with quantized circulation whose value is a material property. Complex tangles of superfluid vortices are often referred to as turbulence [7-9], although this terminology does not imply any direct similarity with the statistical physics of classical vortices in classical fluids like water or air $[10,11]$. Since only a fraction of helium-4 atoms is condensed to form the superfluid, the remaining atoms obey normal-fluid hydrodynamics, and their collective excitations (i.e., phonons and rotons) interact with topological defects via, so-called, mutual-friction forces. The latter play a very important role in finite temperature superfluid turbulence (FTST), where tangles of quantized vortices interact with fully developed, normal-fluid turbulence structures. There are two approaches to FTST that model the effect of topological defects of the order parameter on the hydrodynamics of the normal-fluid: (a) a "coarse-grained" [12-14] approach which refers to scales much larger than the superfluid intervortex spacing, and assumes a continuous superfluid vorticity inter- 
acting with a locally averaged normal-fluid vorticity field. Notably, the governing equations and the parametrization of mutual-friction interactions in this approach are, in principle, flow dependent (e.g., different for rotating and pipe or channel flows), and, by default, not applicable to dilute tangles of a few superfluid vortices, (b) a "direct" approach [15-19] that models discrete topological defects and their individual interactions with the normal-fluid. The purpose of this article is to advance further the direct approach by developing a new model for the coupling between topological defects and normal-fluid hydrodynamics, whose sole empirical input requirement are the standard material properties of the quantum of circulation, superfluid-vortex core radius, and normal-fluid viscosity. Hence, the model is genuinely predictive, and its solutions can (a) directly be compared with experiments which measure local normal-fluid velocities and vortex tangle densities or detect individual superfluid vortices, (b) guide the development of new coarse-grained models [20]. We apply numerical analysis to the new dynamical equations, and solve them algorithmically. In order to calibrate the predictions of the new theory, one solution involves a superfluid vortex ring propagating in a quiescent fluid, and it is compared with similar results produced by employing an older modeling framework. A second solution of the model investigates the effect of two reconnecting normal-fluid vortices (forming initially a Hopf-link) on the structure of ambient superfluid vorticity. A Hopf-link is made of two circles (here vortices) that are linked together exactly once. It is the simplest (nontrivial) link consisting of two components. This is a key interaction that can help understand better fundamental FTST processes. Indeed, previous direct hydrodynamic studies have shown that, although mutual friction forces tend to equilibrate the energy content of the two fluids scale by scale, this is a global (on average) effect that is not valid locally within the flow domain. In other words, the normal-fluid and superfluid vortex structures are locally different from each other. The crucial role of flow instabilities in this phenomenology has been indicated in reference [20]. The new modeling framework will be employed to continue a series of genuinely predictive investigations of superfluid hydrodynamics with topological defects that could directly be compared with analogous experimental findings. 


\section{FINITE TEMPERATURE DYNAMICS OF DISCRETE TOPOLOGICAL DEFECTS}

Our analysis of superfluid vortex dynamics (SFVD) assumes that the latter can be described within the normal-fluid's hydrodynamic framework. Under typical experimental conditions, this is the case for $T>0.8 \mathrm{~K}$. For smaller (yet finite) temperatures a kinetic theory that takes into account quasiparticle interactions (a quantum version of the Boltzmann equation) needs to be employed. For ultra-low temperatures, in the $\operatorname{limit} T \rightarrow 0 \mathrm{~K}$, ballistic quasiparticles could be modelled by the simpler Vlasov equation. In FTST, vortex points $\mathbf{X}_{v}(t)$ comprising a superfluid vortex tangle $\mathcal{L}$ move under the influence of three forces (a) inertial force $\mathbf{f}_{\mathbf{i}}$, (b) Magnus force $\mathbf{f}_{\mathbf{M}}$, and (c) mutual-friction force $\mathbf{f}_{\mathbf{m f}}$ that corresponds to its interaction with the ambient normal-fluid flow. In FTST, the small vortex mass values allow one to neglect $\mathbf{f}_{\mathbf{i}}$ in comparison with the other forces, so $\mathbf{f}_{\mathbf{M}}+\mathbf{f}_{\mathbf{m f}}=0$. We start our analysis by observing the dissipative nature of the Hall-Vinen force [21], a key component of $\mathbf{f}_{\mathbf{m f}}$. This force resembles the drag force on an object moving in a classical fluid, i.e., the Stokes force. Indeed, due to the very small vortex core size (which scatters the normal-fluid quasiparticles), the latter are expected to comprise a creeping flow around the vortices. A second key observation is that a superfluid vortex appears (locally) to the flow similar to a cylindrical rod with circular cross-section. By combining these two observations with low Reynolds number hydrodynamics [22, 23], we can apply to SFVD a known solution of the latter regarding the resistance force on an elongated cylinder (in SFVD a vortex segment) with radius $R$ and length $L$. This force is contained in the plane defined by the cylinder axis and the velocity $\mathbf{v}_{v n}$ of the cylinder/vortex relative to the normal-fluid $\left(\mathbf{v}_{v n} \equiv \dot{\mathbf{X}}_{v}-\mathbf{V}_{n}\right.$, where $\dot{\mathbf{X}}_{v}$ is the vortex velocity $\left(\mathbf{X}_{v}\right.$ is the vortex position in the superfluid vortex tangle), and $\mathbf{V}_{n}$ (referred to as $\mathbf{V}_{n}^{\infty}$ in theory of suspensions) is the asymptotic normal-fluid velocity at the vortex position). $\mathbf{V}_{n}^{\infty}$ is a coarse-grained (possibly turbulent) Navier-Stokes velocity that does not resolve the microscopic (creeping) flow field around the vortex. In SFVD, the cylinder axis is parallel to the tangent vector along the vortex contour, $R$ is equal to the vortex core parameter $\alpha_{0}$, and $L$ is a characteristic vortex dynamical length-scale, which in numerical calculations would be the discretization length $\delta \ell$ along vortices. Core radius $\alpha_{0}$ has a mild variation with temperature around the value $\alpha_{0} \approx 10^{-8} \mathrm{~cm}$, except close to the transition temperature where it diverges. Accordingly, we have two mutual friction 
drag forces per unit length: one normal to the $\mathbf{v}_{v n}$ direction, and another parallel to it: $\mathbf{f}_{D}=D_{\perp} \mathbf{X}_{v}^{\prime} \times\left(\mathbf{X}_{v}^{\prime} \times \mathbf{v}_{v n}\right)+D_{\|} \mathbf{X}_{v}^{\prime}\left(\mathbf{X}_{v}^{\prime} \cdot \mathbf{v}_{v n}\right)$, where the values of $D_{\perp}$ and $D_{\|}$are given by creeping flow analysis: $D_{\perp}=8 \pi \rho_{n} \nu /\left(\ln \left(\xi / \alpha_{0}\right)+0.5\right)$ and $D_{\|}=4 \pi \rho_{n} \nu /\left(\ln \left(\xi / \alpha_{0}\right)-0.72\right)$. $\mathbf{X}_{v}^{\prime}$ is the unit tangent vector at vortex position $\mathbf{X}_{v}$, and $\nu$ is the kinematic viscosity of the normal-fluid. Adding the Iordanskii force [24-26] to $\mathbf{f}_{\mathbf{m f}}$ and the Magnus force, we obtain the following SFVD

$$
\mathbf{X}_{v}^{\prime} \times\left[\rho_{s} \kappa\left(\dot{\mathbf{X}}_{v}-\mathbf{V}_{s}\right)+D_{\perp} \mathbf{X}_{v}^{\prime} \times \mathbf{v}_{v n}+\rho_{n} \kappa \mathbf{v}_{v n}\right]=-D_{\|} \mathbf{X}_{v}^{\prime}\left(\mathbf{X}_{v}^{\prime} \cdot \mathbf{v}_{v n}\right)
$$

where $\kappa$ is the quantum of circulation, and $\rho_{n}$ and $\rho_{s}$ are, correspondingly, the normal-fluid and superfluid mass densities. This equation can only be satisfied if both of its sides are equal to zero, i.e., $\mathbf{X}_{v}^{\prime} \cdot \mathbf{v}_{v n}=0$, and

$$
\rho_{s} \kappa\left(\dot{\mathbf{X}}_{v}-\mathbf{V}_{s}\right)+D_{\perp} \mathbf{X}_{v}^{\prime} \times \mathbf{v}_{v n}+\rho_{n} \kappa \mathbf{v}_{v n}=\lambda \mathbf{X}_{v}^{\prime}
$$

for a real number $\lambda$ to be determined. Solving the last equation in terms of $\dot{\mathbf{X}}_{v}$, employing the decomposition $\mathbf{v}_{v n}=\left(\mathbf{v}_{v n} \cdot \mathbf{X}_{v}^{\prime}\right) \mathbf{X}_{v}^{\prime}-\mathbf{X}_{v}^{\prime} \times\left(\mathbf{X}_{v}^{\prime} \times \mathbf{v}_{v n}\right)$, and using the condition $\mathbf{X}_{v}^{\prime} \cdot \mathbf{v}_{v n}=0$ above, we obtain

$$
\dot{\mathbf{X}}_{v}=\mathbf{V}_{s}-\left(D_{\perp} / \rho_{s} \kappa\right) \mathbf{X}_{v}^{\prime} \times \mathbf{v}_{v n}-\left(\rho_{n} / \rho_{s}\right) \mathbf{v}_{v n}+\left(\lambda / \rho_{s} \kappa\right) \mathbf{X}_{v}^{\prime}
$$

Next, employing the decomposition $\mathbf{V}_{s}=\left(\mathbf{V}_{s} \cdot \mathbf{X}_{v}^{\prime}\right) \mathbf{X}_{v}^{\prime}-\mathbf{X}_{v}^{\prime} \times\left(\mathbf{X}_{v}^{\prime} \times \mathbf{V}_{s}\right)$, multiplying both sides with $\mathbf{X}_{v}^{\prime}$, and demanding that there is no vortex velocity along the tangent, i.e., $\dot{\mathbf{X}}_{v} \cdot \mathbf{X}_{v}^{\prime}=0$, we obtain the following equation for $\lambda: \mathbf{V}_{s} \cdot \mathbf{X}_{v}^{\prime}+\lambda /\left(\rho_{s} \kappa\right)=0$. With this value, the SFVD equation reads

$$
\dot{\mathbf{X}}_{v}=-\mathbf{X}_{v}^{\prime} \times\left(\mathbf{X}_{v}^{\prime} \times \mathbf{V}_{s}\right)-\left(D_{\perp} / \rho_{s} \kappa\right) \mathbf{X}_{v}^{\prime} \times \mathbf{v}_{v n}+\left(\rho_{n} / \rho_{s}\right) \mathbf{X}_{v}^{\prime} \times\left(\mathbf{X}_{v}^{\prime} \times \mathbf{v}_{v n}\right)
$$

Next, we define the coefficients $a=D_{\perp} / \rho_{s} \kappa$ and $b=\rho_{n} / \rho_{s}$, and we rewrite the equation in the form $\dot{\mathbf{X}}_{v}+\mathbf{A} \times \dot{\mathbf{X}}_{v}=\mathbf{B}$, where $\mathbf{A}=a \mathbf{X}_{v}^{\prime} /(1+b)$, and $\mathbf{B}=\left[-\mathbf{X}_{v}^{\prime} \times\left(\mathbf{X}_{v}^{\prime} \times \mathbf{V}_{s}\right)+a \mathbf{X}_{v}^{\prime} \times\right.$ $\left.\mathbf{V}_{n}-b \mathbf{X}_{v}^{\prime} \times\left(\mathbf{X}_{v}^{\prime} \times \mathbf{V}_{n}\right)\right] /(1+b)$. The solution of this equation is [27]

$$
\left.\dot{\mathbf{X}}_{v}=[\mathbf{B}-\mathbf{A} \times \mathbf{B}+(\mathbf{A} \cdot \mathbf{B}) \mathbf{A}] /(1+\mathbf{A} \cdot \mathbf{A})\right]
$$

After straightforward vector algebra, the solution can be written as

$$
\dot{\mathbf{X}}_{v}=-\mathbf{X}_{v}^{\prime} \times\left(\mathbf{X}_{v}^{\prime} \times \mathbf{V}_{s}\right)+\alpha \mathbf{X}_{v}^{\prime} \times\left[\mathbf{X}_{v}^{\prime} \times\left(\mathbf{V}_{n}-\mathbf{V}_{s}\right)\right]+\beta \mathbf{X}_{v}^{\prime} \times\left(\mathbf{V}_{n}-\mathbf{V}_{s}\right),
$$


where $\alpha=-\left[b(1+b)+a^{2}\right] /\left[(1+b)^{2}+a^{2}\right]$, and $\beta=a /\left[(1+b)^{2}+a^{2}\right]$. Notably, $\alpha<0$ and $\beta>0$, hence, the double vector product term contributes to the vortex velocity a part of the component of $\mathbf{V}_{n}-\mathbf{V}_{s}$ that is not along the direction of the vortex tangent, whilst the single vector product term results in the growth of a vortex ring's radius whenever $\mathbf{V}_{n}-\mathbf{V}_{s}$ points along the direction of $\mathbf{V}_{s}$.

Coupling the new SFVD with the Navier-Stokes equation for the normal-fluid [15-19] forms a fully predictive FTST model, bringing superfluid research on par with other areas of hydrodynamics. Hence, we add the conservation of mass in the incompressible normal-fluid $\nabla \cdot \mathbf{V}_{n}=0$, and the conservation of its momentum

$$
\begin{aligned}
& \frac{\partial \mathbf{V}_{n}(\mathbf{x}, t)}{\partial t}+\nabla\left(\frac{p}{\rho_{n}+\rho_{s}}+\frac{\mathbf{V}_{n} \cdot \mathbf{V}_{n}}{2}\right)-\mathbf{V}_{n} \times\left(\nabla \times \mathbf{V}_{n}\right)- \\
& \nu \nabla^{2} \mathbf{V}_{n}-\kappa \int_{\mathcal{L}} d\left|\mathbf{X}_{\mathcal{L}}\right|\left[\mathbf{X}_{\mathcal{L}}^{\prime} \times\left(\mathbf{V}_{n}-\dot{\mathbf{X}}_{\mathcal{L}}\right)\right] \delta^{3}\left(\mathbf{x}-\mathbf{X}_{\mathcal{L}}\right)- \\
& \nu_{c} \int_{\mathcal{L}} d\left|\mathbf{X}_{\mathcal{L}}\right|\left\{\mathbf{X}_{\mathcal{L}}^{\prime} \times\left[\mathbf{X}_{\mathcal{L}}^{\prime} \times\left(\mathbf{V}_{n}-\dot{\mathbf{X}}_{\mathcal{L}}\right)\right]\right\} \delta^{3}\left(\mathbf{x}-\mathbf{X}_{\mathcal{L}}\right)=0,
\end{aligned}
$$

where $p$ is the isotropic part of the normal-fluid pressure tensor, $\mu=\nu \rho_{n}$ is the normal-fluid dynamic viscosity, and $\mathbf{X}_{\mathcal{L}}$ is a position on the superfluid vortex tangle $\mathcal{L}$. Notably, the inertial force acts like a potential force added to the pressure (third term), but also as a nonlinear lift force on vorticity (fourth term or Lamb force), that acts as a generator of turbulence complexity. Although the Lamb force appears to indicate the key role of inertial force on turbulence, one has to be cautious: this term is nonzero only because the viscous stress tensor creates vorticity in the fluid. Turbulence involves a quality of motion initially generated by viscous forces and only subsequently shaped by inertial force. Similarly, although strong small scale strain is a key turbulence feature, it cannot be a feature of any vorticity free inviscid flows. The new form of the mutual-friction coefficient $D_{\perp}$ allows us to define a coupling viscosity $\nu_{c} \equiv D_{\perp} / \rho_{n}$, with $\nu_{c} / \nu=8 \pi /\left(\ln \left(\xi / \alpha_{0}\right)+0.5\right)$. Next we address the obvious question: what are the solutions of this model, and what do they tell us about FTST? We shall see, that, despite the multilayered mathematical complexity of algorithmic solutions, they provide some crucial insights into superfluid physics. 


\section{VORTEX RING PROPAGATING IN A QUIESCENT NORMAL-FLUID}

In order to calibrate the new model, we compare its predictions with a standard computation of a superfluid vortex ring propagating in a quiescent normal-fluid [28]. The numerical methods employed in the calculation are described in [15, 29, 30], which should be consulted for detailed information on the methods and their original sources. The calculation is set within a cubic domain of size $l_{b}=0.1 \mathrm{~cm}$, at $T=1.3 \mathrm{~K}$. The initial normal-fluid velocity is zero. The boundary conditions are periodic. The superfluid ring radius is $R=0.25 l_{b}$, and its initial position is on the centre of the box. On top of the truncation errors of the numerical analysis (as discussed in [15, 29, 30]), the algorithmic calculations introduce round-off errors, since they employ finite precision arithmetic [31] within the set of floating point numbers $\mathbb{F}_{2,53}^{-1022,1023}$, where 2 indicates binary arithmetic, and 53 the precision (significant binary digits). Hence, the distance between 1 and the next larger floating point number is $\epsilon_{m}=0.222 \times 10^{-15}$. The numbers -1022 and 1023 are the powers of 2 that correspond to the smallest and largest numbers that the algorithm arithmetic can represent. In base-10 arithmetic, these numbers are approximately $2.2 \times 10^{-308}$ and $1.8 \times 10^{308}$ correspondingly. The algorithms employ the round to nearest even rounding mode. The numerical grid for the normal-fluid is made of $128^{3}$ grid points. The discretization element along the quantized rings is equal to normal-fluid grid size. The typical time step is $4.365 \times 10^{-5} \mathrm{~s}$.

The results indicate that the new SFVD reproduce two key features: (a) a "double vortex tube" normal-fluid structure (Fig. 1, left) that is induced via mutual-friction excitation, due to the velocity difference between the vortex and normal-fluid velocities, and (b) the characteristic normal-fluid velocity streamlines (Fig. 1, right) generated by a jet-like structure that originates on the vortex contour, as the mutual-friction force "pushes" the normal-fluid there. As in the original calculation, the Reynolds number induced in the normal-fluid is very small $R e \approx 0.284 \times 10^{-1}$. This type of excitations are responsible for generating a complex type of low Reynolds number vortical flow in FTST calculations, that appears in the highest-wavenumbers range of the velocity spectra. In accordance with a visual inspection of new SFVD equations, the calculation predicts the decrease of vortex ring diameter during its propagation. It is nice that the new SFVD does not alter basic physics features in the previous calculations. Moreover its fully predictive character comes together with a direct 

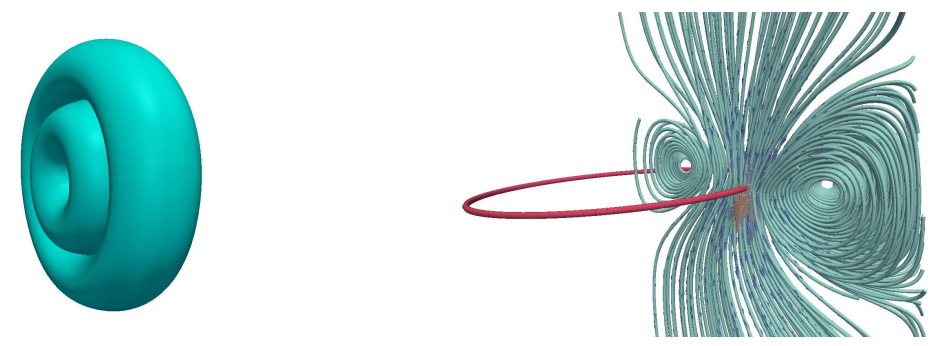

FIG. 1. Superfluid vortex moving in a quiescent normal-fluid. The normalflow field predicted by the new model is similar to that in earlier works [28]. Right: Isocontours of mutual-friction induced normal-fluid vorticity at $t=0.436 \times 10^{-1} \mathrm{~s}$. A dissipative $\left(R e \approx 0.284 \times 10^{-1}\right)$ two vortex tube structure appears. One hundred isocontours spanning the whole range of normal vorticity-magnitude values $\left[\omega_{n}=\left(0.103 \times 10^{-1}-0.370 \times 10^{2}\right) \mathrm{s}^{-1}\right]$ are shown. Left: Normal-fluid velocity streamlines for the same structure. A mutualfriction induced jet at the superfluid vortex position creates two recirculating normal-flow areas adjacent to the superfluid ring contour.

connection between superfluid theory and classical low Reynolds number hydrodynamics.

\section{EFFECTS OF A NORMAL-VORTICITY HOPF-LINK ON THE STRUCTURE OF SUPERFLUID TURBULENCE}

It has previously been shown [18] that a key effect of single normal-fluid vortex ring on superfluid turbulence is the transfer of energy from the normal-fluid to the superfluid towards an equilibration of energies in the two fluids. This however, is not accompanied by the superfluid vorticity mimicking the normal-fluid one. Indeed, it was shown that, under typical conditions, normal-fluid inertia overpowers the mutual-friction force, hence, the latter cannot efficiently correlate superfluid vorticity with the normal-fluid one. In a similar vein, it was shown that, as a result of its interactions with ambient quantized vortices, a straight normal-fluid tube becomes unstable, and only a small percentage of generated superfluid vorticity is eventually trapped by the unstable normal tube mimicking its structure [20]. Instead, the majority of superfluid vorticity propagates in the intervortex space. Here, we introduce the new element of nontrivial vortex topology (a Hopf link), and we investi- 
gate the effect of (reconnection induced) topology change in the normal-fluid on superfluid vortices. We set a calculation within a cubic domain of size $l_{b}=0.1 \mathrm{~cm}$, at $T=1.3 \mathrm{~K}$. The normal-fluid initial conditions are two vortex tubes in a Hopf-link configuration with Reynolds number $R e=\Gamma / \nu=1000$. The individual ring radius $R_{l}=0.125 l_{b}$ (based on the centreline), and the tube radius is $R_{t}=0.2 R_{l}$. The superfluid initial condition is a randomly positioned collection of vortex rings. Two cases were studied: (a) the superfluid rings have diameters similar to the diameters of the normal-fluid rings, and (b) the superfluid rings have an order of magnitude smaller diameter. The boundary conditions are periodic. As mentioned above, the numerical methods are discussed elsewhere [15, 29, 30]. The finite arithmetic precision characteristics of the computations have also been explicated above. The numerical grid for the normal-fluid is made of $128^{3}$ grid points. The discretization element along the quantized rings is equal to normal-fluid grid size. The typical time step is $0.122 \times 10^{-5} \mathrm{~s}$.

\section{THE PURE NORMAL-FLUID HOPF-LINK}

For reference, we include here the evolution of a pure normal-fluid link (Fig. 2). This is well studied in classical fluid dynamics [32-34], and it depicts a dissipative (Fig. 3, left) process via which the original flow helicity (a signature of the topological entanglement of the two tubes [35]) is destroyed (Fig. 3, middle) as a result of intervortex interactions (Fig. 2, middle). Another key physics process is the enstrophy amplification due to vortex stretching during the (relatively) high Reynolds number vortex-tube interactions (Fig. 3, middle). The vorticity isosurface graphic at $t=0.5870 \times 10^{-3} \mathrm{~s}$ (Fig. 2) corresponds to (approximately) the peak in the enstrophy graph.

\section{INTERACTIONS BETWEEN RINGS OF SIMILAR SIZE}

Fig. 4 shows the values of average (normal-fluid) energy, enstrophy and helicity versus time. The pure normal-fluid link data (dotted lines) are also shown for comparison. Due to large normal-fluid inertia, energy flows to the superfluid resulting in smaller normal-fluid energy levels. In agreement with fully resolved turbulence calculations [15], a reduction of 

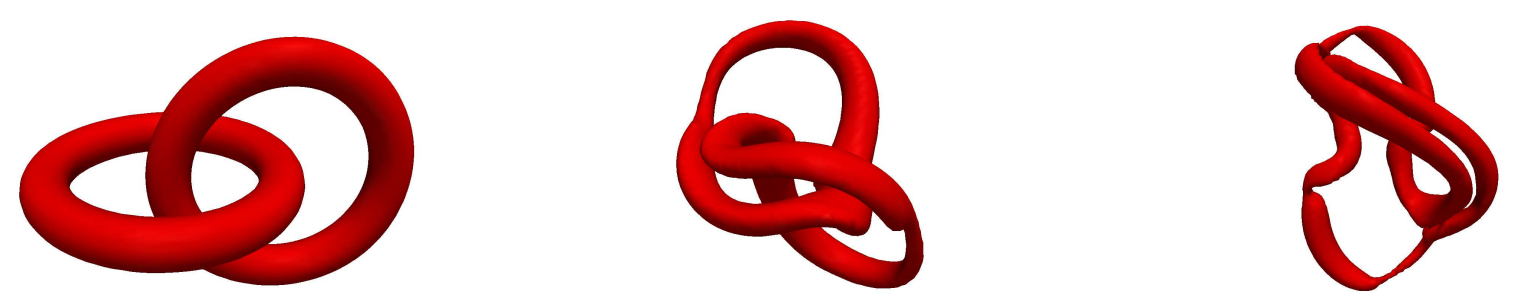

FIG. 2. Pure normal-fluid link. Left: Initial configuration $(t=0)$. Center: Vorticity antialignment process during link evolution $\left(t=0.3088 \times 10^{-3} \mathrm{~s}\right)$. Right: Later stages of the same process $\left(t=0.5870 \times 10^{-3} \mathrm{~s}\right)$. In all cases, vorticity isosurfaces at level $|\boldsymbol{\omega}|=37000 \mathrm{~s}^{-1}$, where $\boldsymbol{\omega}$ is the flow vorticity, are shown. The chosen value corresponds to $30 \%$ of the (global in time) maximum vorticity magnitude. The local variations of tube radii are indicative of vortex stretching intensity.
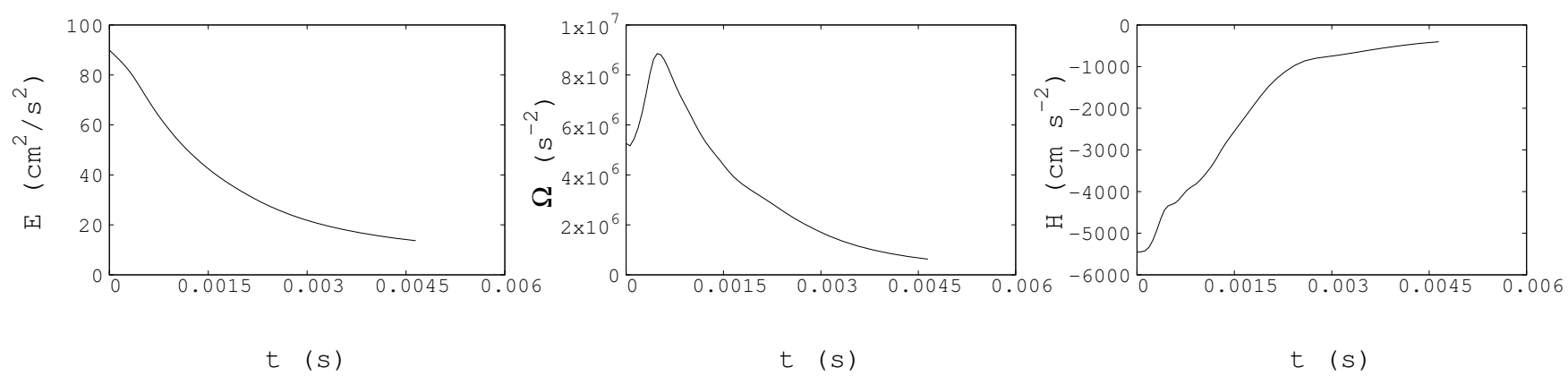

FIG. 3. Pure normal-fluid link. Left: Flow energy $E=(1 / 2 \mathcal{V}) \int d \mathcal{V} \mathbf{V} \cdot \mathbf{V}$ versus time. Center: Flow enstrophy $\Omega=(1 / 2 \mathcal{V}) \int d \mathcal{V} \boldsymbol{\omega} \cdot \boldsymbol{\omega}$ versus time. Right: Flow helicity $H=(1 / 2 \mathcal{V}) \int d \mathcal{V} \boldsymbol{\omega} \cdot \mathbf{V}$ versus time. $\mathcal{V}$ is the volume of the computational box, $\mathbf{V}$ is the flow velocity, and $\boldsymbol{\omega}=\nabla \times \mathbf{V}$ the flow vorticity.

the maximum enstrophy value is observed. In other words, the production of superfluid vorticity dampens intense normal-fluid vortical structures. The later time enstrophy levels that match the pure normal-fluid result are due to generation of normal-fluid enstrophy via mutual-friction excitation at the newly created superfluid ring locations. There is no significant effect on the rate of unlinking of the initial structure (as monitored by the rate of absolute helicity value reduction).

Fig. 5 shows normal-fluid vorticity isosurfaces $\left(|\boldsymbol{\omega}|=37000 \mathrm{~s}^{-1}\right)$ as they coevolve with a dilute tangle of superfluid vortices. In agreement with previous investigations [18], the solution indicates that although the generation sites of superfluid vorticity are somewhat 

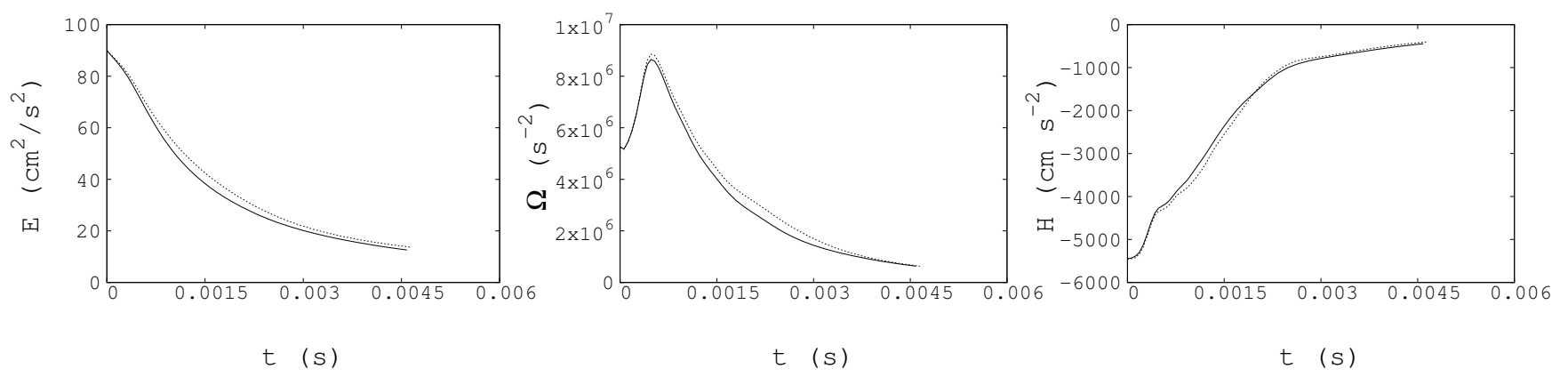

FIG. 4. Normal-fluid Hopf-link interacting with superfluid vortices of similar diameters. Left: Flow energy $E=(1 / 2 \mathcal{V}) \int d \mathcal{V} \mathbf{V} \cdot \mathbf{V}$ versus time. Center: Flow enstrophy $\Omega=(1 / 2 \mathcal{V}) \int d \mathcal{V} \boldsymbol{\omega} \cdot \boldsymbol{\omega}$ versus time. Right: Flow helicity $H=(1 / 2 \mathcal{V}) \int d \mathcal{V} \boldsymbol{\omega} \cdot \mathbf{V}$ versus time. $\mathcal{V}$ is the volume of the computational box, $\mathbf{V}$ is the flow velocity, and $\boldsymbol{\omega}=\nabla \times \mathbf{V}$ the flow vorticity. For comparison, the dotted lines indicate the corresponding pure normal-fluid results.

correlated with intense normal-fluid vorticity locations (the former are located in the neighbourhood of the latter), the formed superfluid structures do not mimic the morphology of the normal-fluid structures, and perform very different motions. This is due to the disparity between normal-fluid and superfluid vortex circulations (that cause the vortices to move with very different self-induced velocities), as well as to the mathematical form and strength of their coupling. The solution indicates a transfer of energy from the normal-fluid to the superfluid in the form of variable-radius, spiral-wave excitations along the superfluid vortex contours. This process is also observed in fully developed FTST solutions [15, 16]. The spiral structures result from the expansion of small-amplitude wiggles that appear on the vortices. The wiggle-formation sites are not correlated with intense normal-fluid vorticity sites. Instead, they are locations where, sufficiently high, axial (i.e., parallel to the superfluid vortex contours) normal-fluid velocities cause the line vortices to become unstable (Fig. 6). The observed instabilities appear similar to the Ostermeier-Glaberson instability [36], that also involves axial normal-fluid flow, and was studied in the context of rotating superfluid flows employing the coarse-grained, Hall two-fluid equations. To illustrate this instability, we have performed a test computation with a uniform normal-fluid velocity of magnitude $V_{n}=150 \mathrm{~cm} \mathrm{~s}^{-1}$ streaming on the plane of a superfluid vortex ring of radius $0.025 \mathrm{~cm}$ (Fig. 7). The results show wiggle formation only on the parts of the superfluid ring that are parallel to the normal-fluid flow. The instability is physical rather than numerical, since there is no sign of the typical zig-zag instability that plagues vortex dynamics calculations 

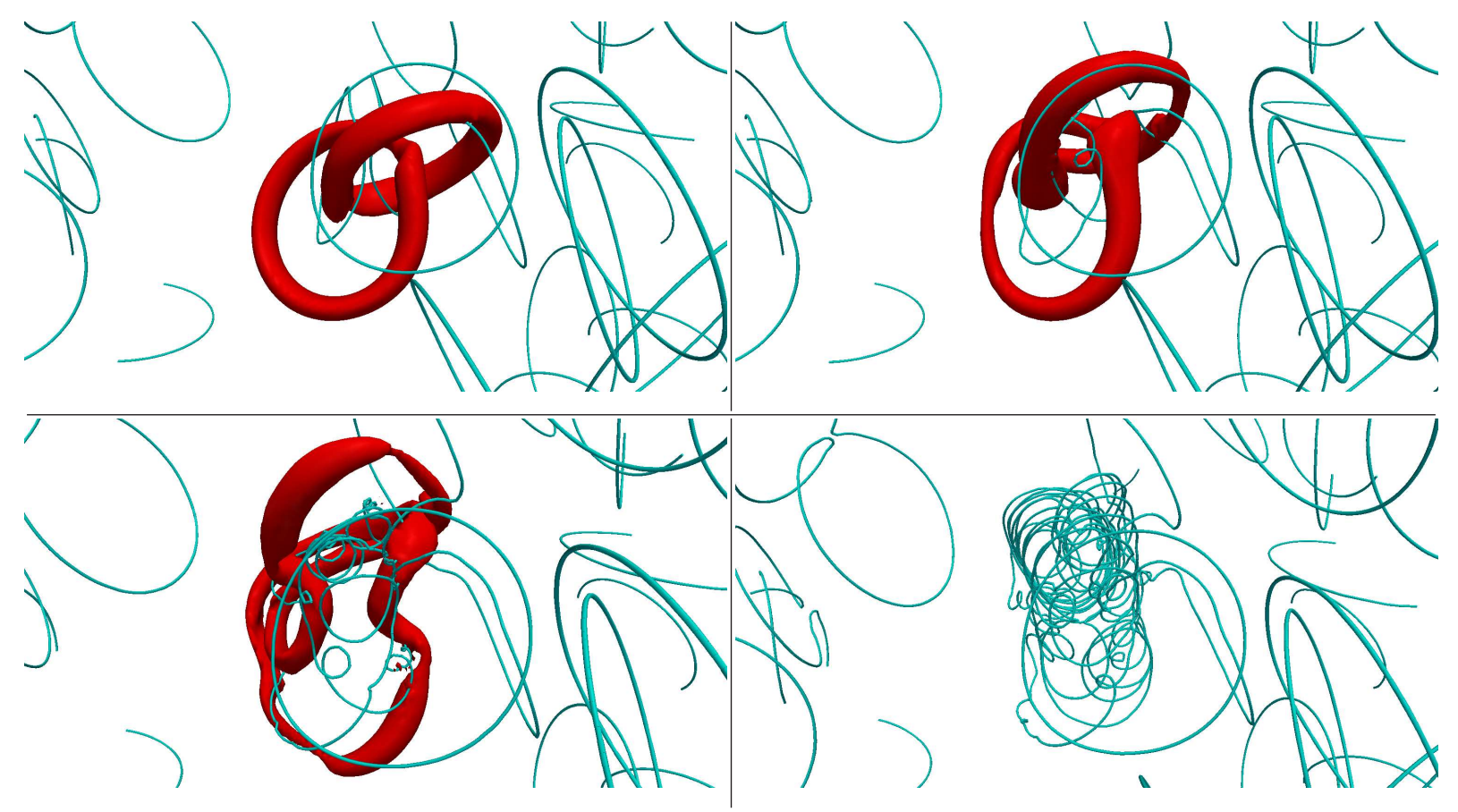

FIG. 5. Normal-fluid Hopf-link interacting with superfluid vortices of similar diameters. Normalfluid vorticity isosurfaces $\left(|\boldsymbol{\omega}|=37000 \mathrm{~s}^{-1}\right)$ and superfluid vorticity configurations. Top left: $t=1.229 \times 10^{-4} \mathrm{~s}$. Significant normal-tube interactions take place without any accompanying important effects on the superfluid tangle. Top right: $t=3.085 \times 10^{-4} \mathrm{~s}$ (before the enstrophy peak). Mutual friction excites superfluid-vortex contour wiggles. Bottom left: $t=5.915 \times 10^{-4} \mathrm{~s}$ (after the enstrophy peak). The superfluid gains energy via wiggle growth processes. Bottom right: $t=2.650 \times 10^{-3} \mathrm{~s}$. There is no normal vorticity isosurface at the prescribed value. In the vortex tangle, some vortices keep growing, whilst other develop wiggle instabilities. The local variations of normal-tube radii are indicative of vortex stretching intensity.

with line vortices, and the instability appears under very different amounts of numerical damping of the Biot-Savart singularity. Moreover, in a second test computation with much smaller normal-fluid velocity $V_{n}=1 \mathrm{~cm} \mathrm{~s}^{-1}$, the ring remained stable, without any signs of wiggle formation.

Another important result (see also [18]) is that the superfluid rings do not grow at sizes larger than the sizes of the energy-providing normal-fluid tubes, as if the normal-fluid vorticity scale determines the upper size of the generated superfluid vorticity structures. This observation is very relevant to the understanding of interscale energy transfers in FTST, and the role of vorticity structures in it. 


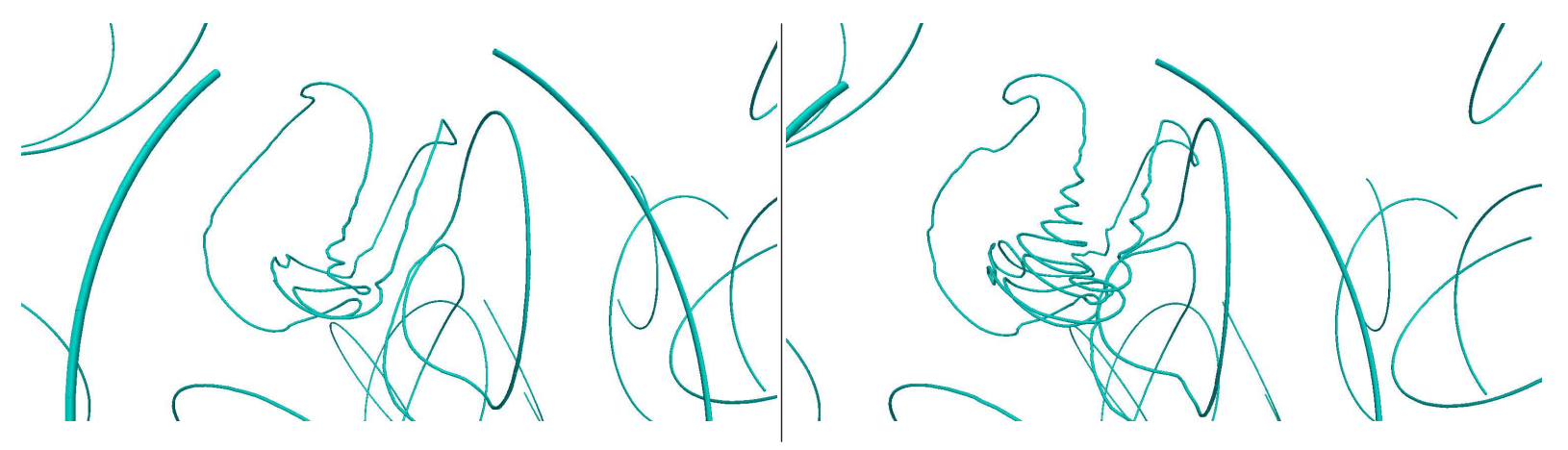

FIG. 6. Normal-fluid Hopf-link interacting with superfluid vortices of similar diameters: mechanism of energy transfer from the normal-fluid to the superfluid. Left: $t=4.794 \times 10^{-4} \mathrm{~s}$ (before the enstrophy peak). Small amplitude wiggles are excited via axial-flow instability on the superfluid vortex at the centre, by normal-fluid velocity pointing (locally) along the vortex direction. Right: $t=5.915 \times 10^{-4} \mathrm{~s}$ (after the enstrophy peak). As energy pours from the normal-fluid to the superfluid, the wiggles grow to form a (variable radius) spiral.
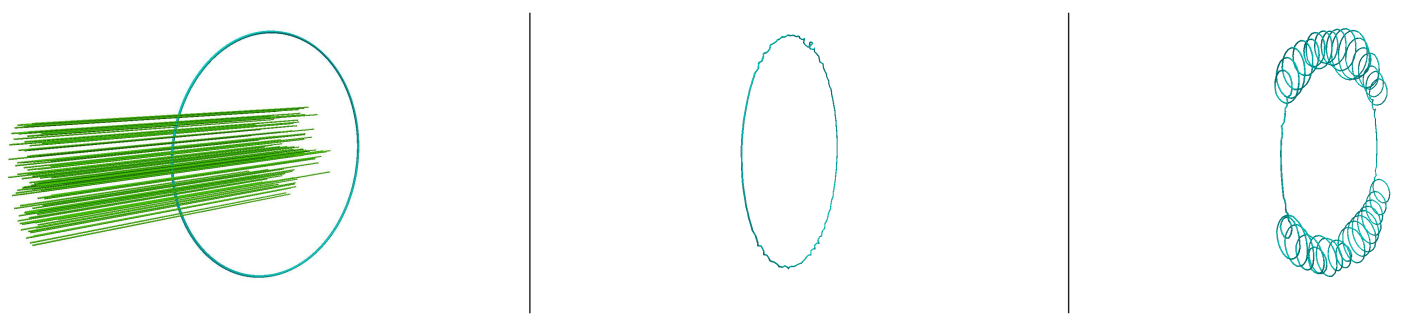

FIG. 7. Superfluid vortex ring moving in a normal-fluid crossflow. Left: Superfluid-vortex ring and sample normal-flow streamlines $(t=0)$. Center: Initiation of wiggle excitation along the ringcontour parts that are parallel with the normal-flow streamlines $\left(t=1.928 \times 10^{-4} \mathrm{~s}\right)$. Right: The wiggles have grown to become spirals that resemble similar structures in the Hopf-link solution $\left(t=3.842 \times 10^{-4} \mathrm{~s}\right)$. It is intriguing that this is an instability caused by the normal-fluid via mutual-friction forcing on a superfluid vortex, whilst reference [20] indicates an instability caused by the superfluid via mutual-friction forcing on a normal-fluid tube.

\section{INTERACTIONS BETWEEN RINGS OF DISPARATE SIZES}

Fig. 8 shows the values of average (normal-fluid) energy, enstrophy and helicity versus time. Since the ratio of the circulations of the two fluids is $\Gamma / \kappa \approx 2337$, and the initial superfluid vortex tangle is dilute, the effect of superfluid vortices on global normal-fluid 

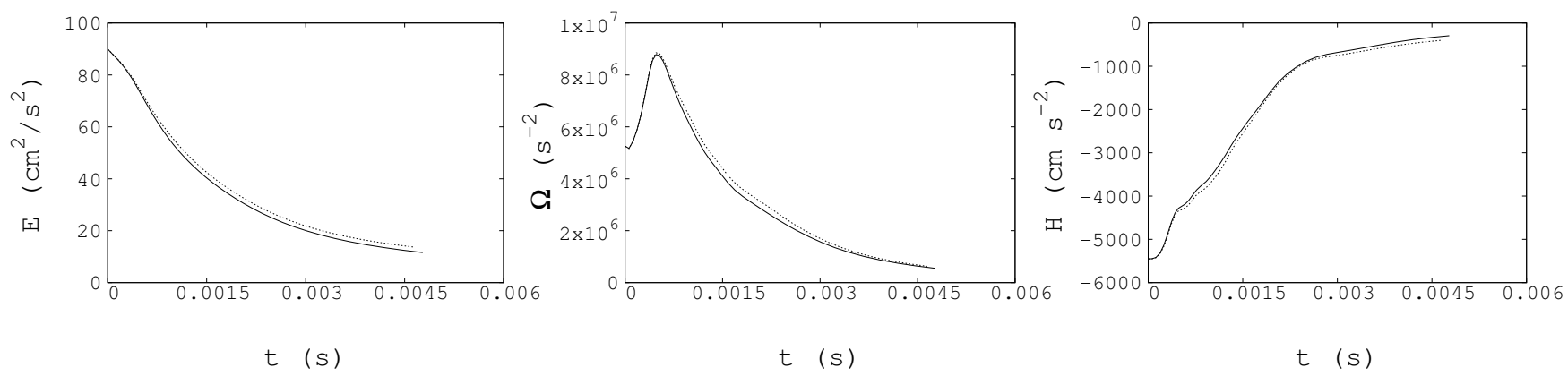

FIG. 8. Normal-fluid Hopf-link interacting with superfluid vortices of much smaller diameters. Left: Flow energy $E=(1 / 2 \mathcal{V}) \int d \mathcal{V} \mathbf{V} \cdot \mathbf{V}$ versus time. Center: Flow enstrophy $\Omega=(1 / 2 \mathcal{V}) \int d \mathcal{V} \boldsymbol{\omega}$. $\boldsymbol{\omega}$ versus time. Right: Flow helicity $H=(1 / 2 \mathcal{V}) \int d \mathcal{V} \boldsymbol{\omega} \cdot \mathbf{V}$ versus time. $\mathcal{V}$ is the volume of the computational box, $\mathbf{V}$ is the flow velocity, and $\boldsymbol{\omega}=\nabla \times \mathbf{V}$ the flow vorticity. For comparison, the dotted lines indicate the corresponding pure normal-fluid results.

quantities is expected to be small. Indeed, only mild variations of the pure normal-fluid solution are observed. Since, after the enstrophy peak, the FTST case enstrophy values are slightly smaller than the corresponding pure normal-fluid values, and the same is true for the energy, we can infer that the reduction in the normal-fluid energy levels is due to a transfer of energy to the superfluid. This is the case, because, the enstrophy dynamics are qualitatively similar to the energy dissipation-rate dynamics, hence since the FTST case dissipation-rate is smaller, only energy transfer could explain the reduction of the energy levels in the FTST case.

Fig. 9 shows normal-fluid vorticity isosurfaces $\left(|\boldsymbol{\omega}|=33000 \mathrm{~s}^{-1}\right)$ as they coevolve with a dilute tangle of superfluid vortices. In comparison with the larger vortex ring case, it is important to note that energy transfer to the superfluid is not associated with vortex instabilities. Instead, the small rings expand to diameters similar to the normal-fluid vortextube diameters whilst remaining smooth. As the normal-vorticity structure evolves, it leaves behind a trail of such expanded rings. The largest-time results (Fig. 9, bottom-right) show that the newly enlarged vortex rings undergo the same instability mechanism that facilitated energy transfer in the larger rings case, thus, indicating that there are two energy transfer mechanisms: ring expansion, and ring wiggle excitation, and subsequent spiral formation. Remarkably, the second mechanism becomes apparent only after the initially small rings have grown to the size of the normal-fluid tubes. Employing the formula for the speed of propagation $V_{\text {wave }}$ of a vortex wave with wavelength $\lambda$ along a vortex contour: $V_{\text {wave }}(\lambda)=$ 


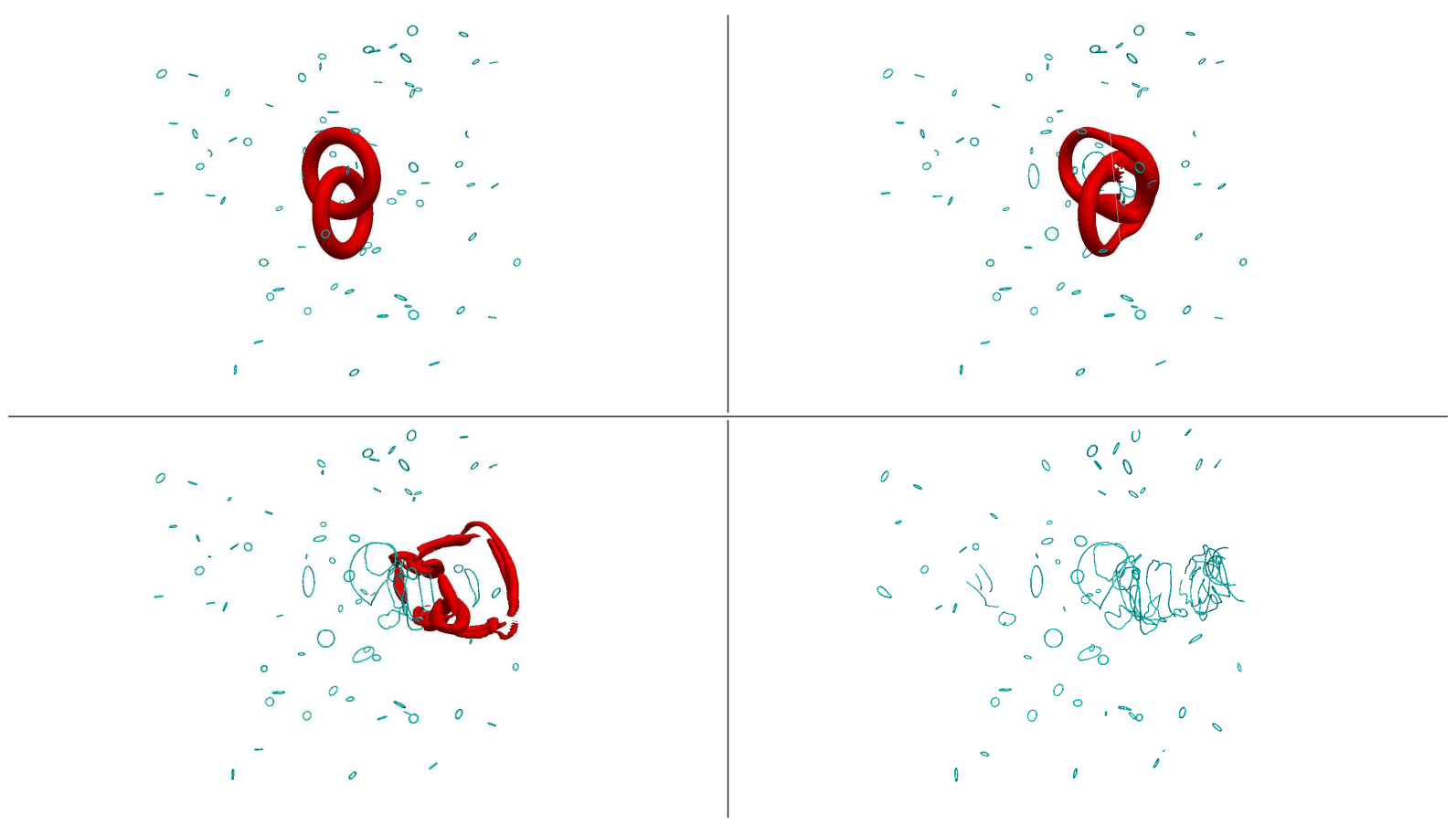

FIG. 9. Normal-fluid Hopf-link interacting with superfluid vortices of much smaller diameters. Normal-fluid vorticity isosurfaces $\left(|\boldsymbol{\omega}|=33000 \mathrm{~s}^{-1}\right)$ and superfluid vorticity configurations. Top left: $t=0$. Top right: $t=3.088 \times 10^{-4} \mathrm{~s}$ (before the enstrophy peak). Some rings have expanded to normal-tube size. Bottom left: $t=1.068 \times 10^{-3} \mathrm{~s}$ (after the enstrophy peak). As the normal-fluid structure evolves, the superfluid gains energy via ring expansion. Bottom right: $t=2.831 \times 10^{-3} \mathrm{~s}$; there is no normal vorticity isosurface at the prescribed value. We observe a tangle of superfluid vortices that trail, the now dissolved, normal-fluid link. Some of the newly augmented rings undergo a similar instability process with the corresponding one of the large rings in the other solution. The local variations of normal tube radii are indicative of vortex stretching intensity.

$\frac{\kappa}{2 \lambda} \ln \left(\frac{\lambda}{2 \pi \alpha_{0}}\right)$, and applying the reasoning of reference [37], we compute that an axial normalfluid velocity of magnitude larger than $V_{n, \|}=1 \mathrm{~cm} \mathrm{~s}^{-1}$ is required to energize waves of wavelength equal to half of a small vortex-loop's length. The average energy data of Fig. 8 suggest that such velocities should be available, so, in principle, spiral-wave formation via axial-flow instability could be observed even for small loops. Hence, they are not observed because either the initial loops are too small for the axial-flow instability to generate wiggles (i.e., we are below the axial-flow instability threshold) or the ring expansion effect irons any possibly excited wiggles out. 


\section{EPILOGUE}

In superfluid helium-4, the order parameter depicts the material flow of an inviscid fluid, and its topological defects correspond to line vortices in this fluid. The helium- 4 atoms that are not described by the order parameter obey normal-fluid hydrodynamics that correspond to the conservation laws of the system. A complete, fully predictive model of normal-fluid hydrodynamics taking into account its coupling with the topological defects of the order parameter has been proposed. It is intriguing, that the normal-fluid becomes aware of the order parameter only via its interaction with topological defects in the latter. Our model exploits another intriguing aspect (brought forward by the pioneering experiments of Hall and Vinen $[38,39])$ : the topological defects appear as obstacles in the normal-flow. Due to the small topological defect sizes, the flow around them can only be a creeping flow, hence, taking into account the linear character of the defects, our model is based on low Reynolds number hydrodynamics around cylindrical objects. When the new defect dynamics is coupled with the Navier-Stokes equations for the normal-fluid, it becomes evident that the coupling between fluid and defects is characterized by two parameters with kinematic viscosity dimensions: the quantum of circulation $\kappa$ in the Iordanskii force term, and a renormalized normal-fluid viscosity $\nu_{c}$ in the Hall-Vinen type force term. The nature of the coupling constants reflects the physics of the corresponding couplings: the Iordanskii force is a consequence of the wave-function dynamics of the normal-fluid at the pre second-quantization level. Indeed, since $\kappa=h / m$, where $h$ is Planck's constant and $m$ is the mass of helium- 4 atoms, $\kappa$ points directly to quantum mechanical effects. On the other hand, the nature of the Hall-Vinen force is very different: the force involves point (quasi)particles that are scattered by the cores of the defects that appear to them as obstacles. This (far from obvious) defect-flow interaction physics is a foundation pillar of our SFVD. So the Iordanskii force is a quantum mechanical force that hints at the fundamental wave-nature of matter and, hence at Planck's constant, whilst the Hall-Vinen force is a (quantum) statistical mechanical force that hints at thermal physics and, hence at fluid viscosity.

We explore the physics of the model by computing the interactions of a normal-fluid vorticity Hopf-link with a dilute suspension of superfluid vortices. The solutions of the model illustrate two different vortex dynamical processes that transfer energy from the normal-fluid 
to the superfluid: (1) an expansion to large sizes of superfluid vortex rings with much smaller diameter than the diameter of the normal-fluid tubes that make up the vortex link. A key observation here is that the superfluid rings do not grow to larger than the normal-fluid tube sizes, (2) a superfluid vortex ring instability due to axial-flow along the vortex contours that excites small amplitude wiggles that subsequently evolve into variable-radius spiral-waves along the vortices. Consistent with the fact that the initially small rings do not expand to larger than the normal-ring sizes, the initially large rings (with diameters similar with the diameters of the Hopf-link tubes) do not receive energy by increasing their size, but only via instability-induced, spiral-wave excitation.

Although the sites of superfluid vorticity generation are always in the neighbourhood of intense normal-fluid vorticity events, the superfluid vortices do not mimic the normal-fluid vorticity structure, and perform very different motions.

Notwithstanding the gained physical insight, the experimental detection of the above vortex dynamical processes presents a challenge. Perhaps the best way for detecting them would be via Particle Tracking Velocimetry (PTV) techniques which employ small particles that do not easily escape superfluid vortices once they collide with them. Since there is significant experience with PTV methods in current superfluid research [40-42], the main difficulty to be encountered in such future experiments is the manipulation of the normalfluid vorticity into organized structures. Such an experimental breakthrough would bring superfluid hydrodynamics on par with more traditional fields in hydrodynamics, enabling a vigorous interaction between theory and experiment. A hopeful case of such an interaction is the thermal counterflow experiment of [41], where PTV measurements indicate significant particle-velocity fluctuations in the streamwise direction even when the normal-fluid is laminar (at low heat fluxes). The creeping flow structures generated by mutual-friction forces at the superfluid vortex locations, as indicated here and in reference [28], could very well be responsible for the observed particle-velocity fluctuations. 


\section{ACKNOWLEDGEMENTS}

I am very grateful to Tony Leonard for valuable discussions, and Marco Ghiani for help with the graphics.

[1] M. Schlosshauer, Decoherence: And the Quantum-To-Classical Transition, Berlin: Springer (2010).

[2] A. Wasserman, Thermal Physics: Concepts and Practice, Cambridge: Cambridge University Press (2011).

[3] J. Casalderrey-Solana, H. Liu, D. Mateos, K. Rajagopal, U. A. Wiedemann, Gauge/String Duality, Hot QCD and Heavy Ion Collisions, Cambridge: Cambridge University Press (2014).

[4] D. Forster, Hydrodynamic Fluctuations, Broken Symmetry, And Correlation Functions, Boca Raton: CRC Press (1995).

[5] O. Wiese, D. Marenduzzo, O. Henrich, Microfluidic flow of cholesteric liquid crystals, Soft Matter 12, 9223, (2016).

[6] O. Henrich, K. Stratford, D. Marenduzzo, P.V. Coveney, M.E. Cates, Confined cubic blue phases under shear, J. Phys.: Condens. Mat. 24, 284127 (2012).

[7] S.K. Nemirovskii, Quantum turbulence: Theoretical and numerical problems, Physics Reports 524, 85-202 (2013).

[8] C.F. Barenghi, R.J. Donnelly (eds.), Quantized Vortex Dynamics and Superfluid Turbulence, Berlin: Springer (2010).

[9] R.J. Donnelly, Quantized Vortices in Helium II, Cambridge: Cambridge University Press (2008).

[10] P.A. Davidson, turbulence, Oxford: Oxford University Press (2004).

[11] A. Tsinober, An Informal Introduction to Turbulence, Kluwer Academic Publishers (2001).

[12] L. Biferale, D. Khomenko, V. Lvov, A. Pomyalov, I. Procaccia, G. Sahoo, Turbulent statistics and intermittency enhancement in coflowing superfluid He-4, Phys. Rev. Fluids 3, 024605 (2018).

[13] K.W. Schwarz, Three-dimensional vortex dynamics in superfluid ${ }^{4} H e$ : Line-line and lineboundary interactions, Phys. Rev. B 31, 5782 (1985). 
[14] D. Jou, M.S. Mongiovi, M. Sciacca, Hydrodynamic equations of anisotropic, polarized and inhomogeneous superfluid vortex tangles, Physica D 240, 249 (2011).

[15] D. Kivotides, Energy spectra of finite temperature superfluid helium-4 turbulence, Phys. Fluids 26, 105105 (2014).

[16] D. Kivotides, Spreading of superfluid vorticity clouds in normal-fluid turbulence, J. Fluid Mech. 668, 58 (2011).

[17] D. Kivotides, Decay of finite temperature superfluid helium-4 turbulence, J. Low Temp. Phys. 181, 68 (2015).

[18] D. Kivotides, Interactions between normal-fluid and superfluid vortex rings in helium-4, EPL 112, 36005 (2015).

[19] S.K. Nemirovskii, Energy spectrum of the 3D Velocity Field, Induced by Vortex Tangle, J. Low Temp. Phys. 171, 504 (2013).

[20] D. Kivotides, Mutual-friction induced instability of normal-fluid vortex tubes in superfluid helium-4, Phys. Lett. A 382, 1481 (2018).

[21] C.F. Barenghi, R.J. Donnelly, W.F. Vinen, Friction on quantized vortices in Helium II. A Review, J. Low Temp. Phys. 52, 189 (1983).

[22] E. Guyon, J.-P. Hulin, L. Petit, C.D. Mitescu, Physical Hydrodynamics, Oxford: Oxford University Press (2015).

[23] P. Oswald, Rheophysics: The Deformation and Flow of Matter, Cambridge: Cambridge University Press (2009).

[24] G. E. Volovik, Three nondissipative forces on a moving vortex line in superfluids and superconductors, Pis'ma v ZhETF 62, 58 (1995).

[25] G. E. Volovik, Vortex versus spinning string: Iordanskii force and gravitational AharonovBohm effect, Pis'ma v ZhETF 67, 841 (1998).

[26] L. Thompson, P.C.E. Stamp, Quantum dynamics of a Bose superfluid vortex, Phys. Rev. Lett. 108184501 (2012).

[27] O. C. Idowu, D. Kivotides, C. F. Barenghi, D. C. Samuels, Equation for Self-Consistent Superfluid Vortex Line Dynamics J. Low Temp. Phys. 120, 269 (2000).

[28] D. Kivotides, C.F. Barenghi, D.C. Samuels, Triple vortex ring structure in superfluid helium II, Science 290, 777 (2000). 
[29] D. Kivotides, Relaxation of superfluid vortex bundles via energy transfer to the normal fluid, Phys. Rev. B 76, 054503 (2007).

[30] D. Kivotides, Interactions between vortex tubes and magnetic-flux rings at high kinetic and magnetic Reynolds numbers, Phys. Rev. Fluids 3, 033701 (2018).

[31] W. Tucker, Validated Numerics: A Short Introduction to Rigorous Computations, Princeton: Princeton University Press (2011).

[32] H. Aref, I. Zawadzki, Linking of vortex lines, Nature 354, 50 (1991).

[33] D. Kivotides, A. Leonard, Computational model of vortex reconnection, Europhys. Lett. 63, 354 (2003).

[34] M. Brady, A. Leonard, D. I. Pullin, Regularized Vortex Sheet Evolution in Three Dimensions, J. Comp. Phys. 146, 520 (1998).

[35] V. Arnold, B. Khesin, Topological Methods in Hydrodynamics, Berlin: Springer (1998).

[36] R.M. Ostermeier, W.I. Glaberson, Instability of vortex lines in the presence of axial normal fluid flow, J. Low Temp. Phys. 21, 191 (1975).

[37] D.C. Samuels, D. Kivotides, A damping length scale for superfluid turbulence, Phys. Rev. Lett. 83, 5306 (1999).

[38] W.F. Vinen, Mutual friction in a heat current in liquid helium II. II. Experiments on transient effects, Proc. R. Soc. London, Ser. A 240, 128 (1957).

[39] W.F. Vinen, Mutual friction in a heat current in liquid helium II. III. Theory of the mutual friction, Proc. R. Soc. London, Ser. A 242, 493 (1957).

[40] A.I. Golov, P.M. Walmsley, P.A. Tompsett, Charged Tangles of Quantized Vortices in Superfluid 4He, J. Low Temp. Phys. 161, 509 (2010).

[41] B. Mastracci, W. Guo, An exploration of thermal counterflow in He II using particle tracking velocimetry, Phys. Rev. Fluids 3, 063304 (2018).

[42] M. La Mantia, P. Svankara, D. Duda, and L. Skrbek, Small-scale universality of particle dynamics in quantum turbulence, Phys. Rev. B 94, 184512 (2016). 